\title{
Erratum: Lithium-induced Cardiotoxicity: A Rare Clinical Entity
}

Sudeep Acharya ${ }^{1}$, Abdul Hasan Siddiqui ${ }^{2}$, Shamsuddin Anwar ${ }^{1}$, Saad Habib ${ }^{1}$, Rabih Maroun 3

1. Internal Medicine, Staten Island University Hospital, Staten Island, USA 2. Pulmonary and Critical Care Medicine, University of Illinois Urbana Champaign, Champaign, USA 3. Internal Medicine, Staten Island University Hospital, Staten Island , USA

Corresponding author: Shamsuddin Anwar, shamsduhs15@gmail.com

Corresponding author: Shamsuddin Anwar

1. Internal Medicine, Staten Island University Hospital, Northwell Health, Staten Island, USA 2. Pulmonary and Critical Care Medicine, University of Illinois at Urbana-Champaign, Champaign, USA 3. Internal Medicine, Staten Island University Hospital Northwell Health, Staten Island, USA 4. Internal Medicine, Staten Island University Hospital, Staten Island, USA 5. Internal Medicine, Staten Island University Hospital, Staten Island, USA

\section{How to cite this erratum}

Acharya S, Siddiqui A, Anwar S, et al. (August 06, 2020) Correction: Lithium-induced Cardiotoxicity: A Rare Clinical Entity. Cureus 12(8): c33. doi:10.7759/cureus.c33

\section{Correction Notice}

Due to a technical error, the name of the third author, Shamsuddin Anwar, was incorrectly repeated as the fifth author. The fifth author should have been listed as Rabih Maroun. The authors have been updated accordingly. Cureus sincerely regrets the error. 